\title{
Stage IIIB Lung Cancer AJCC v8
}

National Cancer Institute

\section{Source}

National Cancer Institute. Stage IIIB Lung Cancer AJCC v8. NCI Thesaurus. Code C136480.

Stage IIIB includes: (T1a, N3, M0); (T 1b, N3, M0); (T 1c, N3, M0); (T 2a, N3, M0); (T2b, N3, M0); (T3, N2, M0); (T4, N2, M0). T1a: Tumor measuring $1 \mathrm{~cm}$ or less in greatest dimension. A superficial, spreading tumor of any size whose invasive component is limited to the bronchial wall and may extend proximal to the main bronchus also is classified as T1a, but these tumors are uncommon. T1 b: Tumor measuring more than 1 $\mathrm{cm}$ but $2 \mathrm{~cm}$ or less in greatest dimension. T1 $\mathrm{c}$ : Tumor measuring more than $2 \mathrm{~cm}$ but 3 $\mathrm{cm}$ or less in greatest dimension. T2a: Tumor measuring more than $3 \mathrm{~cm}$ but $4 \mathrm{~cm}$ or less in greatest dimension. T2b: Tumor measuring more than $4 \mathrm{~cm}$ but $5 \mathrm{~cm}$ or less in greatest dimension. T3: Tumor measuring more than $5 \mathrm{~cm}$ but $7 \mathrm{~cm}$ or less in greatest dimension or directly invading any of the following: parietal pleura (PL3), chest wall (including superior sulcus tumors), phrenic nerve, parietal pericardium; or separate tumor nodule(s) in the same lobe as the primary. T4: Tumor measuring more than $7 \mathrm{~cm}$ or tumor of any size invading one or more of the following: diaphragm, mediastinum, heart, great vessels, trachea, recurrent laryngeal nerve, esophagus, vertebral body, or carina; separate tumor nodule(s) in an ipsilateral lobe different from that of the primary. N2: Metastasis in ipsilateral mediastinal and/or subcarinal lymph node(s). N3: Metastasis in contralateral mediastinal, contralateral hilar, ipsilateral or contralateral scalene, or supraclavicular lymph node(s). M0: No distant metastasis. (AJCC 8th ed.) 